\title{
Designing Research Prototype for the Elderly: a Case Study
}

\begin{abstract}
This paper describes a research study regarding intergenerational story sharing of the elderly living in the nursing home, including four iterations, applying a Research-through-Design approach. It started from an exploration prototype named Interactive Gallery (1st iteration), and its findings helped to narrow down our research area and define our research question. To answer it, the prototype named Slots-story (2nd iteration) and Slots-memento (3rd iteration) were designed and implemented, which focused on life story and memento story of the elderly respectively. While the 4th iteration aimed at facilitating intergenerational story sharing and sustainably. The above research iterations offer an example of how research prototypes supports to focus research area, and answer the research question in stages. We finally conclude with a discussion of insights on designing prototype for the non-tech-savvy elderly.
\end{abstract}

\section{Keywords}

Research-Through-Design, Research Prototype, Elderly, Storytelling, Tangible Interface

\section{Introduction}

The aging society is coming. The worldwide population over age 65 is expected to more than double from 357 million in 1990 to 761 million in 2025, and with up to $50 \%$ of those over the age of 85 likely to be placed in a nursing home at some point in their lives [1]. However, social isolation is widespread among older adults in nursing homes, and older residents have limited involvement in social connections [2]. Living separately with their children also makes it difficult for younger and older family members to communicate with each other because of different interests, stereotypes of aging, geographical distance, and the fast pace of contemporary life. Since social interaction of the elderly is a broad research area, our research focuses on its subset: intergenerational storytelling of the elderly living in nursing homes.

Given that one of the most precious characteristics of older adults is their memory of events, people and places [3], intergenerational storytelling could act as an effective way to keep them stay in touch with their children. Our target group is the aged non-tech-savvy people living in a nursing home. While younger seniors are embracing online social technologies, the Internet and social media use drop off significantly for people age 75 and older-Only 34\% of people in the G.I. Generation (born in 1936 or earlier) use the Internet, and $21 \%$ have home broadband [4]. 
In this paper, we describe our three-year research study of intergenerational story sharing of the elderly, in a Research-through-Design manner. It offers an example of how research prototypes support us to focus research area, answer research question in stages. We then present our insights on designing research prototype for the non-tech-savvy older adults.

\section{Related Work}

Social technology for the elderly. Existing research indicates that their social interaction could be promoted either by strengthening connections between older adults and their existing social circles (friends, family, etc.), or expanding their social circle by knowing more friends [5]. Since TUI (Tangible user interface) has been identified as having great potential to improve older adults' acceptance of technology acceptance [6], related applications for non-tech-savvy older adults mostly adopt tangible interface.

Research-through-Design. R-t-D is described in the literature as an approach for scientific inquiry, taking advantage of the unique insights gained through design practice [7]. Although the term of R-t-D is not a new concept, it is until recent years that it has been widely discussed and used in the $\mathrm{HCl}$ field, and became an increasingly recognized approach in design. R-t-D is conceptualizing research done using the skillful practice of design activity, revealing research insights [8]. One of the features lies in that it acknowledges and embraces professional practices' contributions to knowledge[9].

Research Prototype. R-t-D also highlights the importance of research prototype. The use of designedly prototype within the research process has been well acknowledged. Prototypes are defined as research instruments created by the researchers, tailored to each individual study [10]. Prototypes serve multiple functions within the research process: Prototypes make abstract theory concrete as they could involve people in research process, and they are 'like products' in the sense that someone can interact with them and experience them [11]. Prototypes are also seen as embodying designers' judgments about valid ways to address the possibilities.

Summary. Our research focuses on the storytelling between older residents in the nursing home and their children. The older adults are story producers, while their children are the memory trigger producers. $R-t-D$ and research prototype have been widely discussed and used in $\mathrm{HCl}$ field, but there are few research that specifically discuss the application study of R-t-D for the elderly people. In the next section, we explain how our R-t-D cycles evolve in detail.

\section{Iterative Design Cycles}

The research question of our project is: How can we use design to facilitate intergenerational storytelling and preservation for older adults living in the nursing home? To answer this question, three sub-questions are formulated: (1) What are their stories about? (2) In which ways, can design facilitate them to tell and preserve stories? (3) In which ways, can design involve their children? The entire research project, including the above research question, was done in a Research-through-Design manner.

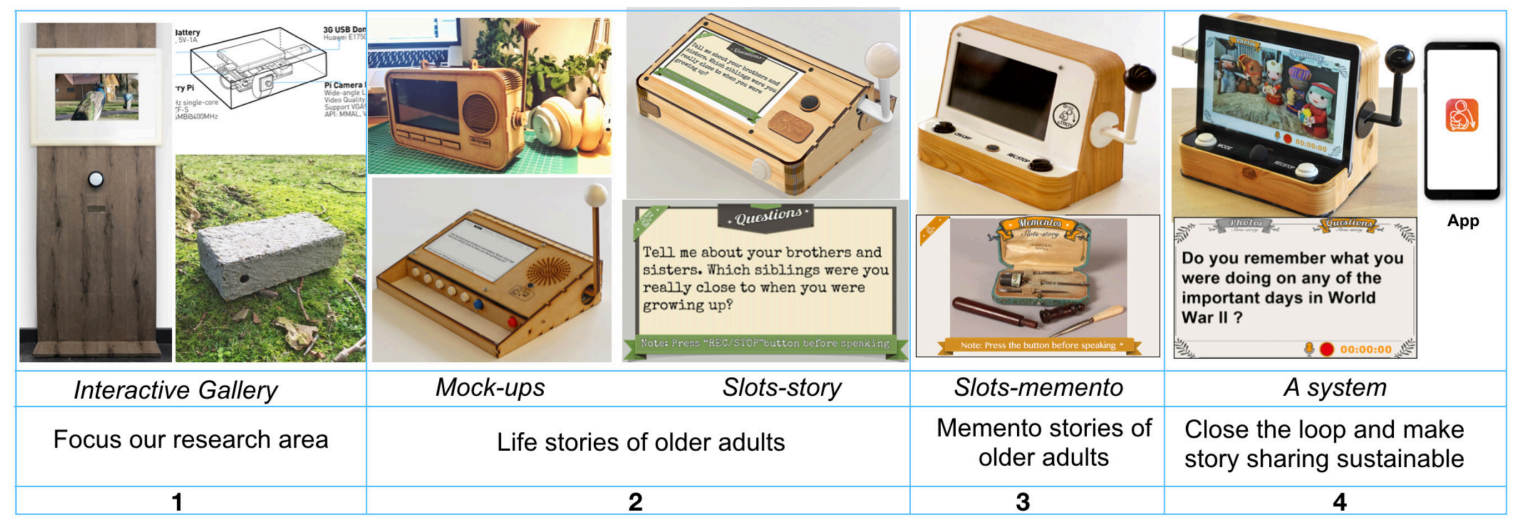

Fig. 1. Overview of our research iterations 


\subsection{First Prototype: Focus Our Research (Define RQ)}

As social interaction of the elderly is a broad and macroscopic research area, our first prototype Interactive Gallery was an explorative prototype, designed to uncover the related potential factors, narrow down research goal and define the research question. Details process could be seen in [12], and the following is a short description.

Contextual inquiry. We firstly conducted semistructured interviews with seven older adults and two caregivers in a Dutch nursing home. We found older adults encountered difficulties in connecting with their fellow residents. The nursing home was not a fully open community, which made it a relatively isolated and independent. Most of them couldn't operate digital devices, and they still highly relied on physical operation.

Prototype. In response to this, we designed the Interactive Gallery system (Figure 1-(1)), which consisted of a set of scenery-collectors and a gallery-like interactive installation. The formers are distributed to volunteers from local communities to share real-time scenery photos with older adults, and the latter is placed in the nursing home which enables older adults to watch and start conversations. Communication between sharers and receivers will also be connected through a "postcard-sending" metaphor: The older adults could print scenery photos as postcards by simply pressing the button. They could choose to send back to sharers, or keep them.

Field Study. It was implemented in a Dutch nursing home for five weeks. Direct observation method (including baseline observation and intervention observation) were conducted, and semi-structured interviews were contacted semi-structured interviews with 13 older adults (ranged in age from 71 to 86 ).

Finding. The field study proved the tangible interface employing metaphor reduces using barriers for them. Their memories were evoked by the familiar scenery photos. They preferred to share memories with their children, rather than the unacquainted volunteers.

The above finding inspired us that the elderly could be deemed as content (memory, story, etc.) producers, and their children were the story listeners. This drove us to narrow down our research goal and further identify our research question.

\subsection{Second Iteration: the Elderly's Life Stories (Answer rq1,rq2)}

The following is a brief description, and a detailed process could be seen in [13]. We firstly conducted semi-structured interviews with both the older adults and their children, and the following design requirements were defined: Memory trigger. Tangible interface employing metaphor. Using audio as the storage medium of stories. Could be used either face-to-face or separately by older adults and their children. Then three concepts were built based on the design requirements and were developed to be mock-ups(Figure1-(2)). Older adults were consulted to evaluate them. Slots-story employing metaphor of slots-machine was chosen and further detailed. Slotsstory, a slots machine-like device, aims to facilitate inter-generational life story sharing and preservation. It utilizes with the metaphor of slots machine, and integrates functions of memory cue generator, story recording, and preservation. By default, there are 40 trigger questions covering most aspects of an entire life. It could either be used face-to-face or separately by older adults and their children. In the field study, eight pairs of participants from a Dutch nursing home were recruited to use the prototype for around ten days, and each pair consisted of an old adult (six female and three male, ranged in age from 77 to 89) and his/her child. Semi-structured interviews were conducted with both older adults and the young. Stories were transcribed and analyzed.

Reflection: As some stories were related to their mementos, such as album, souvenir, etc., which were also ideal memory triggers as they provided visual clues. This inspired us to explore their mementos and related stories in the next iteration. Additionally, the appearance of porotype should be refined according to older adults' feedback.

\subsection{Third Iteration: Their Memento Stories} (Answer rq1,rq2)

Research Prototype. We interviewed older and young adults separately. We first asked them to arrange a brief guided tour of their homes, aiming to 
examine their mementos for displaying and stored in hidden places. The prototype was refined based on the interview and feedback of the 2 nd iteration: Prototype's decorative effects needed to be highlighted to make it unobtrusive when putting it at older adults' home (Figure1-(3)). It was used in a cross-generational operation manner: the young took photos of older adults' mementos and copied them to the prototype, while older adults used the prototype to tell stories related to the mementos [14].

Field Study. Ten pairs of participants (each pair consisted of an old adult and child) were recruited to use it for around seven days, and mementos and recordings were collected. Semi-structured interviews were conducted with older adults and their children. Mementos were categorized and analyzed. Stories were firstly transcribed, then were analyzed.

Reflection. Sustainability of the intergenerational sharing was necessary. Next, a cellphone application will be designed for the young side.

\subsection{Fourth Iteration: Involve Their Children (Answer rq3)}

Aim. In this iteration, we close the intergenerational storytelling loop by designing an App for the young generation, aiming to facilitate intergenerational story sharing and preservation in a sustainable manner.

Prototype. A system consisting of a slot machine-like device used by older adults, a cellphone application used by the young. It integrates life story and memento story sharing (Figure 2)[15].

\section{Discussion}

We have illustrated our R-t-D cycles including four research prototypes, which provide insights on how $\mathrm{R}-\mathrm{t}-\mathrm{D}$ is applied to research practice, especially for the elderly people. In this section, we present the implications in two parts: Prototype as tools of identifying and answering research question, and designing research prototypes for the non-tech-savvy elderly.

\subsection{Prototype as Tools for Identifying and Answering Research Question}

\section{Prototype Acts as a Means of Narrowing Down}

Research Focus. First, Design research is a way to ask larger questions beyond the limited scope of a design problem, through the practice of design itself.

Our research focus and detailed research question were identified through the first prototype Interactive Gallery. As mentioned, social interaction of the elderly is a broad and macroscopic research area: their social connections with fellow residents, with family members, old friends, people from the local community, caregivers, .etc. Interactive Gallery was firstly implemented, and its findings helped us to focus our research on intergenerational storytelling of older adults

(Section 3.1). Next, new and unexpected findings emerged during the implementation of prototype, which guided our follow-up research. Our second prototype was regarding life stories of the elderly. However, new and unexpected findings emerged during its implementation: some stories were related to family

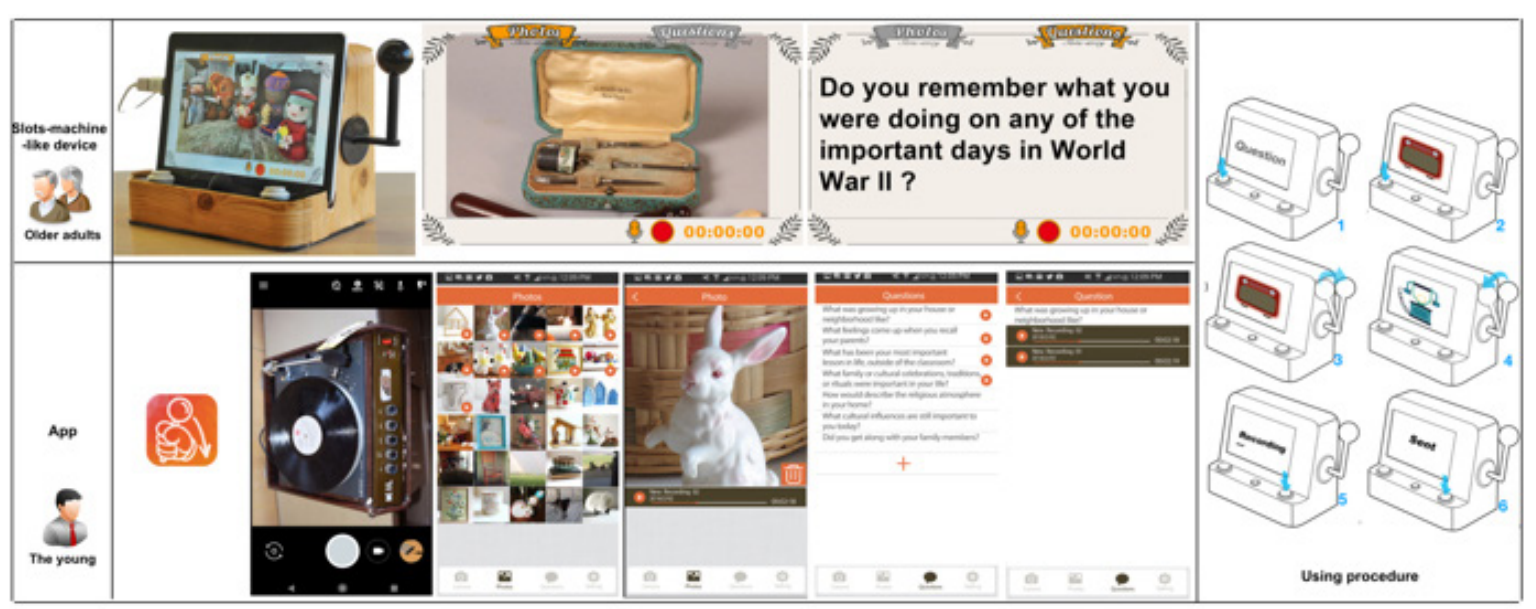

Fig. 2. Prototype of 4th iteration 

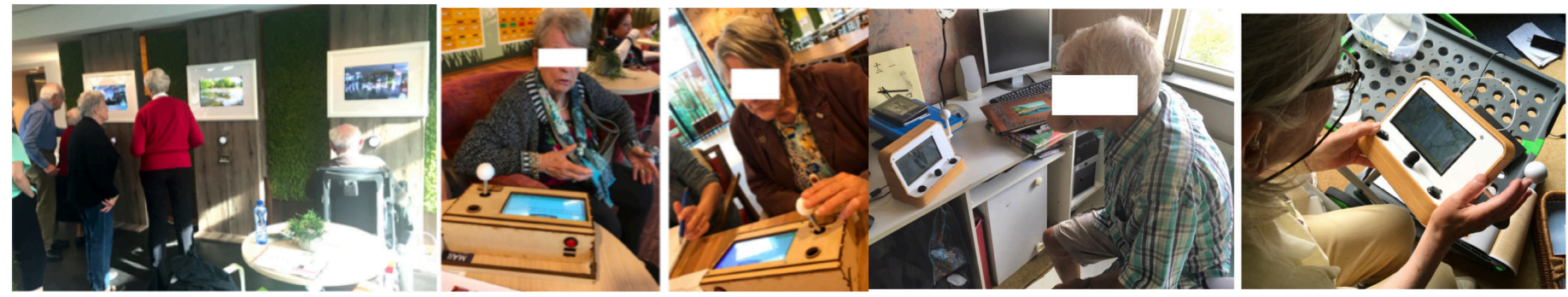

Fig. 3. Interview with older adults in different iterations mementos, such as album, souvenir, artwork, etc. This inspired us to explore their mementos and related stories in the next iteration (Section 3.3).

\section{Answering Research Question in Stages through} the Evolution of Prototypes. Our overall research question was answered in stages. RtD itself has highly iterative character as the design evolves while conducting RtD-led research [16]. As mentioned, we break the overall research question into three subquestions. To answer rq1 and rq2, we designed and implemented the 2 nd and the 3 rd prototype, which focused on older adults' life stories and memento stories respectively. To answer rq3, we designed the 4th prototype, to fully involve the older adults' children. The evolution of prototypes is not only a response to the development of research questions and insights, but also the feedback of the participants. For example, the appearance of the 3rd porotype was refined based on the feedback in 2 nd iteration, as the elderly thought it should be unobtrusive when putting it at home, therefore decorative and vintage effects needed to be highlighted.

During the research process, we switched between reflection and practice. Knowledge is gained by conducting a design exercise and continuously reflecting on direct and indirect observations, beliefs and experiences[16]. In our R-t-D cycles, reflection is the output of the implementation of the current prototype, also the input for the next prototype. Therefore, reflection is the dividing line between analyzing and practice: it is the end of a phase of study, but the beginning of a new phase of study. The reflection is a catalyst for knowledge generation.

\subsection{Designing Research Prototypes for the Non- Tech-Savvy Elderly}

Non-tech-savvy Older Adults. One thing to note is that, since people above the age of 65 years old are diverse regarding cognitive ability, they compromise a group that is considerably more diverse than people of the general (younger) population. As such, the experience of using technology of each individual older person is unique, and their level of technological mastery varies. Therefore, they could be roughly divided into the non-tech-savvy and tech-savvy group. Our target group is older adults in nursing home, according to literature and our investigation, most of them are non-tech-savvy users.

The following are some insights on designing prototypes for the non-tech-savvy elderly derived from our research practices. Since all of our prototypes adopted tangible interface, the presentation of our insights is partly based on Eva Hornecker et al's framework on tangible interaction. The framework is structured around four themes: Tangible Manipulation, Spatial Interaction, Embodied Facilitation, and Expressive Representation. Each theme concludes serval concepts[17].

\section{Providing Haptic Direct Manipulation through a Tangible Interface.}

It has been well acknowledged that tangible interfaces are more accessible and suitable for the needs of elderly people, as they could provide a natural style of interaction [1]. The elderly are suffered from declines in motor control and accuracy, while physical contact with an interface gives elderly users confidence in their abilities[18]. Current touch-based interfaces are mainly visually guided without physical feedbacks. This lack of tangible feedback often leads to several errors and frustrations that are accentuated in older people. Haptic direct manipulation refers to tactile contact, haptic feedback, and material qualities when manipulating the 
interaction objects[17]. All our research porotypes adopted tangible interfaces providing haptic direct manipulation. Interactive Gallery was equipped with big buttons, and the elderly could manipulate it by a simple hand-press. Slots-story and Slots-Memento provided tangible interfaces and intuitive interactions through the lever. Both operations were accepted by the elderly users according to our field study. As one older adult said in our interview: "Its function is simple. I especially like the lever, and the knob is easy to hold."

\section{Providing Representational Significance through}

Metaphor. Representational significance refers to the interrelation of physical and digital representations, and how users perceive them[17]. It is important to communicate information with analogies and metaphors that are relatable to the systems that older adults are familiar with, and a new product that is designed in familiar metaphors can reduce the barriers of elderly users to use [14]. Metaphors of the gallery, postcardsending, and slots-machine were applied our prototypes respectively. The interaction styles were based on the elderly's familiar knowledge to help them understand easily. During our interview of the first iteration, one older adult said: "It's like a photo art gallery, and it is better because the photos are dynamic." For our second iteration, one older adult said: "The slots-machine-like operation raises me a sense of expecting and curiosity for the unknown."

Providing Paper Instructional Manual. As our prototypes were used by the elderly independently, the instructional manual was needed to tell them how to operate the prototypes. During our interview of the first iteration, one older adult said: "It is too novel for me, I dare not use it." Another said: "Actually I dare not to press the button and I ask others or my son to print postcard for me." Research also indicated the elderly have reported that they are afraid of using new technological devices as they are afraid of damage the device [19]. Therefore, in the 2nd iteration, we provided the elderly participants with paper instructional manuals, which consisted of step-by-step instructions and corresponding illustrations (Figure 4). The paper manual could also afford easy annotation, which was the elderly were generally more familiar with, compared with online content. During our study, we found older adults in the nursing home still relied on newspaper to get information. The instructions in paper manuals are static, which are easier for some older users, as it matched their learning style. Research indicates that older adults have a stronger preference for using the device's instruction manual over trial-and-error because it matches their learning style.

\section{Tailoring Interface to the Elderly: Aesthetic and}

Visibility. User interfaces need to be tailored to the specific user group-the elderly. Two aspects were considered in our case, aesthetic and visibility.

Firstly, according to our study, elderly people were still interested in traditional physical objects, vintage and old-fashioned were in line with the aesthetic view of the elderly. Therefore, the vintage style was applied in the appearance of the Interactive Gallery. While in the interview with the elderly regarding the appearance of Slots-Story, its decorative and vintage effects needed to be highlighted as they hoped prototype could be unobtrusive when putting it at home. As one older adult said during the interview of the 2 nd iteration: "I think I have enough house appliances in my home, I don't want another one." "I don't use digital devices, and I think the vintage style could bring a sense of mysteriousness." "The vintage style could give indications of past things." Secondly, big and bold fonts need to be adopted considering fading the eyesight of the elderly. The capability of the eye to focus on near objects is diminishing for the elderly. The visual presentation of information should consist of large text, big and clear buttons. During our interview,

Fig. 4. The paper instructional manual of Slots-story

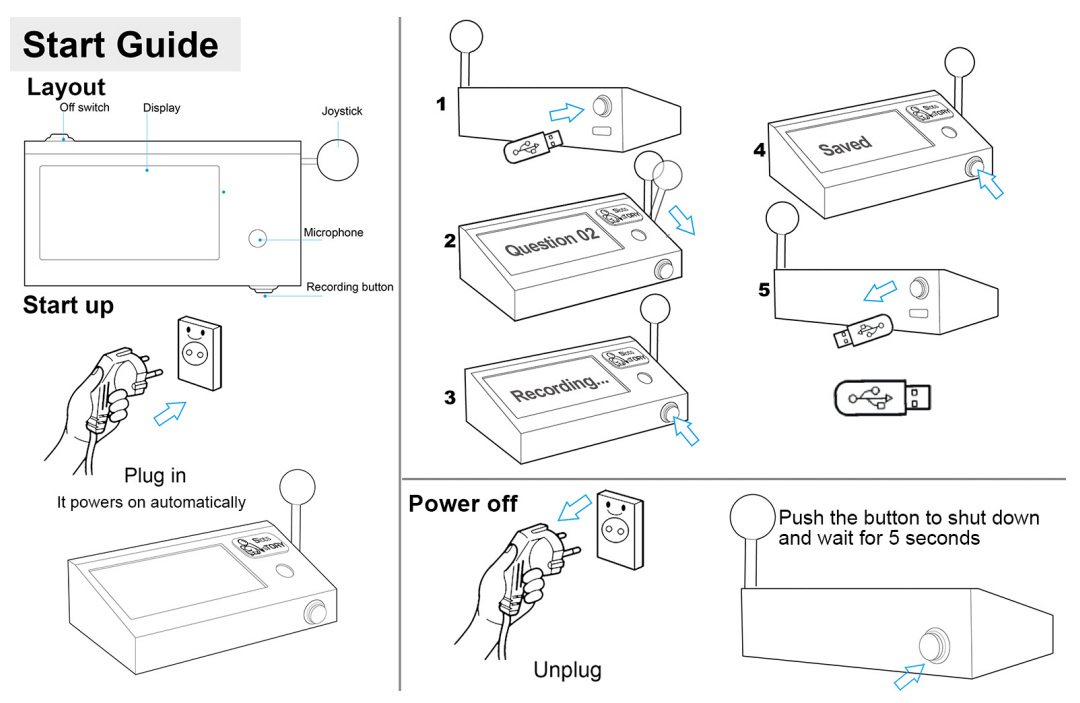


the older adults were suffering from a decreased vision: "Two things that have great influences on my life, bad mobility and bad eyesight."

Avoid Accurate Operation. Large movements rather than delicate operations were applied in our prototypes. The elderly are suffering from the decline in fine motor skills and accuracy of movements, which makes it harder to use small buttons and switches. In our case, big button and lever were designed in Interactive Gallery and Slots-story respectively, to compensate for the elderly suffering from the decline in the accuracy of movements. As one older adult said during our interview: "I used to listen to radios, and I still have one in my room. But I feel difficult to operate precisely when I scroll through stations by turning a knob." Some older adults mentioned the sensitivity of the Slots-story's operation should be reduced, as their hands were clumsy.

\section{Providing Embodied Constraints through Minimization of the Number of Interface} Components. Embodied constraints refer to the physical set-up constraining users' behaviors. This restriction eliminates the possibility of making errors, and eliminating the possibility of errors could lower the anxiety brings to the elderly. Our experiences of providing Embodied constraints is minimizing the number of interface components: In Interactive Gallery, there was only one interface component--a big button. While in Slots-story, there were only two operation components: a handle and a group of buttons. During our interview, older adults appreciated the simple interaction.

\section{Conclusion}

In this paper, we report our research study regarding intergenerational story sharing of the non-tech-savvy elderly people living in nursing homes, applying R-t-D. We first discuss the research prototype as tools of identifying and answering the research question, including prototype acting as means of narrowing down research focus, answering research question in stages through the evolution of prototypes, and switching between reflection and practice. These lessons have a universal significance for R-t-D application study. We then present our lessons of designing research prototypes for the non-tech-savvy elderly, including providing Haptic direct manipulation through tangible interface, providing Representational significance through metaphor, providing paper instructional manual, tailoring interface to the elderly: aesthetic and visibility, avoid accurate operation, and providing Embodied constraints through minimization the number of interface components.

\section{References}

1. Reichman, W. E., Coyne, A. C., Borson, S., Negrón, A. E., Rovner, B. W., Pelchat, R. J., ... Hamer, R. M. (1998). Psychiatric consultation in the nursing home: a survey of six states. The American Journal of Geriatric Psychiatry, 6(4), 320-327.

2. Mor, V., Branco, K., Fleishman, J., Hawes, C., Phillips, C., Morris, J., \& Fries, B. (1995). The Structure of Social Engagement among Nursing Home Residents. The Journals of Gerontology Series B: Psychological Sciences and Social Sciences, 50B(1), P1-P8. doi:10.1093/geronb/50B.1.P1

3. Dryjanska, L. (2015). A social psychological approach to cultural heritage: memories of the elderly inhabitants of Rome. Journal of Heritage Tourism, 10(1), 38-56. doi:10.108 0/1743873X.2014.940960

4. Madden, M., Lenhart, A., Cortesi, S., \& Gasser, U. (2010). Pew Internet and American life project. Washington, DC: Pew Research Center

5. Waycott, J., Vetere, F., Pedell, S., Kulik, L., Ozanne, E., Gruner, A., \& Downs, J. (2013). Older adults as digital content producers. In Proceedings of the SIGCHI Conference on Human Factors in Computing Systems (pp. 39-48). ACM. Retrieved from http://dl.acm.org/citation.cfm?id=2470662

6. Spreicer, W. (2011). Tangible interfaces as a chance for higher technology acceptance by the elderly. In Proceedings of the 12th International Conference on Computer Systems and Technologies (pp. 311-316). ACM.

7. Frayling, C. (1993). Research in art and design.

8. Zimmerman, J., Stolterman, E., \& Forlizzi, J. (2010). An analysis and critique of Research through Design: towards a formalization of a research approach. In Proceedings of the 8th ACM Conference on Designing Interactive Systems (pp. 310-319). ACM.

9. Godin, D., \& Zahedi, M. (2014). Aspects of research through design: a literature review. Proceedings of DRS, 1667-1680.

10. Boer, L., Donovan, J., \& Buur, J. (2013). Challenging industry conceptions with provotypes. CoDesign, 9(2), 73-89.

11. Soegaard, M., \& Dam, R. F. (2012). The encyclopedia of 
human-computer interaction. The Encyclopedia of Human-

Computer Interaction.

12. Li, C., Lin, X., Kang, K., Hu, J., Hengeveld, B., Hummels,

C., \& Rauterberg, M. (2018). Interactive Gallery: Enhance Social Interaction for Elders by Story Sharing. In $M$.

loannides, J. Martins, R. Žarnić, \& V. Lim (Eds.), Advances

in Digital Cultural Heritage (pp. 104-116). Cham: Springer International Publishing.

13. Li, C., Hu, J., Hengeveld, B., \& Hummels, C. (2018). Slotsstory: facilitate inter-generational life story sharing and preservation of the elderly. In Proceedings of the 10th Nordic Conference on Human-Computer Interaction (pp. 691-695). ACM

14. Li, C., Hu, J., Hengeveld, B., \& Hummels, C. (2019). Slots-Memento: Facilitating Intergenerational Memento Storytelling and Preservation for the Elderly. In Proceedings of the Thirteenth International Conference on Tangible, Embedded, and Embodied Interaction (pp. 359-366). New York, NY, USA: ACM. doi:10.1145/3294109.3300979

15. Li, C. (2019). Designing a System to Facilitate Intergenerational Story Sharing and Preservation for Older Adults. In Proceedings of the Thirteenth International Conference on Tangible, Embedded, and Embodied Interaction (pp. 737-740). ACM.

16. Reeker, L. F., \& Brazier, F. M. T. (n.d.). Lessons Learned from Research through Design, 7.

17. Hornecker, E., \& Buur, J. (2006). Getting a grip on tangible interaction: a framework on physical space and social interaction. In Proceedings of the SIGCHI conference on Human Factors in computing systems (pp. 437-446). ACM. Retrieved from http://dl.acm.org/citation.cfm?id=1124838

18. Häikiö, J., Wallin, A., Isomursu, M., Ailisto, H., Matinmikko, T., \& Huomo, T. (2007). Touch-based user interface for elderly users. In Proceedings of the 9th international conference on Human computer interaction with mobile devices and services (pp. 289-296). ACM. Retrieved from http://dl.acm. org/citation.cfm?id=1378021

19. Laguna, K., \& Babcock, R. L. (1997). Computer anxiety in young and older adults: Implications for human-computer interactions in older populations. Computers in human behavior, 13(3), 317-326. 